\title{
Limitations in attending to a feature value for overriding stimulus-driven interference
}

\author{
TAKATSUNE KUMADA \\ National Institute of Bioscience and Human-Technology, Ibaraki, Japan
}

\begin{abstract}
Six experiments were conducted to examine the effect of knowledge of a target for overriding stimulus-driven interference in simple search tasks (Experiments 1-3) and compound search tasks (Experiments 4-6). In simple search when the target differed from nontargets in orientation, a singleton distractor that had an orientation equivalent to that of a target interfered with search for the target. When the singleton distractor was less salient than the target with respect to the target-defining feature, it still caused interference. Such within-dimensional, nonsaliency-based interference also occurred in compound search tasks. In contrast, no interference occurred when a singleton distractor was defined in cross-dimension in a simple search task. When a compound search task was used, the salient distractor interfered with the search for a less salient target. These results are discussed in terms of their applicability to existing models and the limitations of top-down penetrability of a feature processing stage.
\end{abstract}

A salient object presented in a visual field can be detected without subjective impression of any attentional scrutiny. This phenomenon, referred to as pop-out, has been extensively examined using visual search tasks (Treisman, 1988; Treisman \& Gelade, 1980; Treisman \& Gormican, 1988). Pop-out is experimentally defined as the shallow slope (less than 5 or $6 \mathrm{msec} / \mathrm{element}$ ) of the search time function for detecting a target as a function of the number of distractors (Treisman \& Souther, 1985). Generally, pop-out is observed when the target is defined against distractors by one feature difference, such as color, orientation, brightness, shape, or size (e.g., Treisman, 1988; Treisman \& Gelade, 1980). It is argued that pop-out is mediated by preattentive or automatic coding of visual features that are applied in parallel across the visual field. This parallel feature processing serves as the basis for guiding focal attention to a particular region of the visual field (e.g., Cave \& Wolfe, 1990; Koch \& Ullman, 1985; Wolfe, 1994).

In recent studies, the mechanism of shifts of focal attention induced by a feature singleton during a visual search task has been investigated (e.g., Theeuwes, 1994; Yantis \& Hillstrom, 1994). The effects of the presentation of a feature singleton distractor, which are explained by assuming that attention is captured by the feature singleton, are discussed in terms of the locus of two ways of attentional control-namely, goal-directed control and stimulus-driven control. Goal-directed control is mediated by an observer's knowledge of the target, whereas stimulus-

The author thanks W. Bacon, C. Folk, T. Kikuchi, J. Theeuwes, and $S$. Yantis for their helpful comments on earlier versions of this paper. Correspondence should be addressed to T. Kumada, Human Informatics Department, National Institute of Bioscience and Human-Technology, 1-1 Higashi, Tsukuba, Ibaraki 305-8566, Japan (e-mail: kumada@nibh. go.jp). driven control is based on bottom-up activation that is computed in parallel feature processes. Several studies suggest that the two ways of control are not independent but are interactive or competitive (Folk, Remington, \& Johnston, 1993; Yantis, 1993).

Several studies have revealed the strong effect of stimulus-driven control of attention. Presentation of some kind of feature singleton in a visual search display causes a distracting effect of searching for a target irrespective of the observer's goal in the task. First, a singleton distractor that is more salient than the target interferes with the search for the target even when the singleton distractor is defined with respect to an irrelevant feature dimension (Theeuwes, 1991a, 1992, 1994). Second, dynamic visual changes, such as abrupt visual onsets, can interfere with the search for a target (Yantis \& Johnson, 1990; Yantis \& Jones, 1991; Yantis \& Jonides, 1990).

With respect to the effect of top-down control on the stimulus-driven control of attention, experimental conditions in which the extent of interference from the feature singleton is reduced by prior knowledge of a target have been explored. First, knowledge of the target location is very effective in overriding stimulus-driven interference from a feature singleton (Theeuwes, 1991b; Yantis \& Jonides, 1990). Second, when subjects know a particular feature value of the target and the feature singleton is irrelevant to the target, goal-directed selection could override stimulus-driven interference (Bacon \& Egeth, 1994; Pashler, 1988). In Experiment 6 of Pashler's study, although no standard visual search task was used, irrelevant color variation did not interfere with the search for a form singleton when subjects knew the form of the target. In contrast, Theeuwes (1992) reported that the presence of a salient singleton with respect to an irrelevant feature dimension interfered with the search for a target, even when subjects knew the feature value of the target. This result 
suggested that goal-directed selection based on a particular feature value could not be used for overriding stimulusdriven interference from the irrelevant singleton.

Bacon and Egeth (1994) explained these results by assuming two modes of top-down processing: the feature search mode and the singleton detection mode. They argued that the subjects in the study conducted by Theeuwes (1992) might have used the singleton detection mode, in which subjects set their attention to detect any singletons on the visual display. This assumption is consistent with the data-driven selection model proposed by Theeuwes (1991a, 1992, 1994). In that model, spatial attention is involuntarily captured by the most salient singleton in the visual field. If the most salient singleton is not the target, attention is assumed to be automatically switched to the next most salient singleton. Therefore, when subjects adopt the singleton detection mode, distraction by the most salient singleton is inevitable regardless of the knowledge of the target-defining feature value.

On the other hand, Bacon and Egeth (1994) assumed that Pashler's (1988) subjects adopted the feature search mode: Subjects voluntarily monitor a specific feature map that codes for the presence of a relevant feature. If the irrelevant singleton does not activate the monitored feature map, it does not interfere with the detection of the target. They examined the validity of this assumption by using tasks similar to those employed by Theeuwes (1991a, 1992). When subjects were forced to search for a target feature, not for a singleton, there was no distracting effect of an irrelevant singleton distractor. They concluded that goal-directed selection of a specific known feature value could be used for overriding stimulus-driven interference from a salient feature singleton.

It is worthwhile to examine the conclusion more closely. Bacon and Egeth (1994), in their Experiment 3, presented one or two nontargets that had a unique feature value with respect to a target-defining feature (i.e., shape) for the purpose of forcing subjects to search for a specific target feature value (i.e., circle), but not for the most salient singleton. In some trials, a distractor that was different in color from other display elements was presented. They found no effect of the distractor but a significant effect of the number of nontargets with unique forms. When subjects were forced to search for a specific form, the presentation of a color singleton distractor did not interfere with the detection of the form-defined target. The results were considered as evidence in favor of the feature search mode. Since the subjects could confine their search to a specific feature value, an irrelevant distractor that had a unique feature value with respect to another feature dimension did not interfere with the detection of the target.

Bacon and Egeth interpreted the significant effect of the number of nontargets with unique forms, in which the reaction times (RTs) increased as a function of the number of nontargets with unique forms, as the withinnontarget heterogeneity effect in visual search (Duncan \& Humphreys, 1989). However, another interpretation of the result is also possible. The significant effect of the number of nontargets with unique forms could be interpreted as the effect of attentional capture by the unique nontargets. The RTs were prolonged as a result of incorrect attentional capture by nontargets that had a unique feature value with respect to the target-defining feature. If this interpretation were accepted, the results would show that the subjects could not confine their search to a prespecified target feature value. Therefore, the new interpretation leads to a hypothesis that attentional control settings could be tuned to a specific feature dimension, not to a specific feature value. The subjects could not attend to a specific feature value, even when they knew the target feature value.

The results of Experiment 6 of Pashler (1988) are also not considered to be direct evidence that subjects can attend to a particular feature value that defines a target. Bacon and Egeth (1994) interpreted those results as evidence that an irrelevant singleton interferes substantially only when the target feature is unspecified. In other words, the singleton does not interfere with the detection of a target when the target feature is known. Since the task involved localization of a singleton target, it could be solved by singleton detection within a target-defining feature dimension. Therefore, it was not necessary for the subjects to use their knowledge of a particular target feature value. The result can also be explained by assuming that attentional control settings could not be tuned to a specific feature value. That is, in the study, the subjects could override the interference from an irrelevant feature singleton by attending to the specific feature dimension.

Recent studies have proposed a mechanism of top-down control of a specific feature dimension (Found \& Müller, 1996; Müller, Heller, \& Ziegler, 1995). Müller and his colleagues proposed a dimension-weighting mechanism that serves to weight a specific feature dimension when a target feature is known. They proposed dimensionspecific saliency maps. The total activation of each of the stimulus elements was computed in terms of the weighted sum of individual dimension-specific saliency maps. When the target feature is known, the feature dimension is assigned a large weight. In the model, the feature dimension of a singleton target must be known to obtain a pop-out response, but particular target values for that dimension are not necessary. However, the mechanism was not assumed when the subjects were required to attend to a specific feature value. Therefore, it is still not clear whether top-down control can be used for overriding interference from a singleton distractor that is unique with respect to the target-defining feature.

This study was primarily concerned with attentional selectivity among salient elements that are defined by a single feature dimension. Recently, Folk and Remington (1998) examined the within-dimensional selectivity using a spatial cuing paradigm. They found that an irrelevant feature singleton captures spatial attention only when the singleton is defined by the same feature value as the target. On the other hand, previous studies using visual search tasks have suggested that subjects can con- 
fine their search to a specific feature dimension (Bacon \& Egeth, 1994; Müller et al., 1995; Pashler, 1988). However, there is no direct evidence that subjects can attend to a specific feature value to override interference from a singleton distractor in the context of visual search studies.

The purpose of this study was to investigate the efficiency of knowledge of a target feature value for overriding stimulus-driven interference, using a visual search task. Consider a typical visual search display in which a target differs from uniform distractors, referred to as nontargets in this article, only in orientation. Previous research suggested that the target pops out when it is sufficiently different from nontargets (e.g., Treisman \& Gelade, 1980). Next, assume that the visual search display also contains one distractor that differs from nontargets in the same dimension as the target (i.e., orientation in this case) but that has a feature value different from the target with respect to the target-defining feature; this distractor also pops out in the display if it is used as a target. Such a distractor is referred to as a singleton distractor in this article. The questions addressed in this study were whether the singleton distractor defined with respect to the target-defining feature interferes with the detection of the target and whether the interference effect can be observed when the roles of the target and the singleton distractor are reversed.

Under these display conditions, two patterns of results are predicted from two different models, which correspond to two modes of processing as proposed by Bacon and Egeth (1994). The first model assumes that there is a complete attentional control setting for a target-defining feature value. Since the target feature value is known and is constant in all the trials, and since subjects can use their knowledge of the target feature value to search for the target, the singleton distractor will not interfere with the detection of the target. Therefore, this model predicts that no interference effect of the singleton distractor on the detection of the target will be observed. The second model assumes that subjects cannot use their knowledge of the target feature value to search for a specified target. This is considered to apply to within-dimensional selection in the data-driven selection model (Theeuwes, 1991a, 1992), although Theeuwes proposed this model on the basis of cross-dimensional interference using separable visual feature dimensions. It is predicted from this model that performance of target search is determined by the relative saliency of the target and the singleton distractor irrespective of prior knowledge of the target. If the target is less salient than the singleton distractor, the singleton distractor interferes with the detection of the target. However, if the target is more salient than the singleton distractor, the singleton distractor does not interfere with the detection of the target. Therefore, this model predicts an asymmetric, or one-way, interference effect of a singleton distractor on the detection of a target.

This study was basically modeled after previous studies on attentional capture (Bacon \& Egeth, 1994; Theeuwes, 1991a, 1992), with two major modifications. First, in this study, a simple visual search task was used in addition to a compound search task. Previous studies used different tasks; although a compound visual search task has been widely used in studies of attentional capture (e.g., Bacon \& Egeth, 1994; Theeuwes, 1991a, 1992), Müller and his colleagues used a simple visual search task (Found \& Müller, 1996; Müller et al., 1995). The compound search task was useful for demonstrating the limitations in processing the reported feature in the second, limitedcapacity stage of visual processing (Duncan, 1985). However, the discussion became complicated when the compound search task was used, because many recent findings and models obtained using the simple visual search task cannot be simply applied to the results obtained using the compound search task. Indeed. Theeuwes (1991a) recognized a difference between the compound search task used in his study and the simple visual search task. He noted that a focal attentional process was always required in compound search tasks because the reported feature could not be detected without focal attention on the target. However, he argued that, in the simple visual search task, the subjects could respond on the basis of the detection of activity in a particular feature map, thereby bypassing the focal attentional process. To discuss the results of the recent models obtained from the use of simple visual search tasks, simple feature search tasks (Experiments 1-3) and compound search tasks (Experiments 4-6) should be used with similar stimulus displays in the same study.

Second, instead of shape dimension, which was used in previous studies (Bacon \& Egeth, 1994; Theeuwes, 1991a, 1992), orientation dimension was used as a targetdefining feature. Using shape dimension, it is difficult to compare the saliency of one value (e.g., a circle) with that of another (e.g., a triangle) among backgrounds (e.g., diamonds). In contrast, the saliency of two elements that differ in the orientation dimension can be manipulated and described on the basis of the angular difference between the two elements (see also Wolfe \& Friedman-Hill, 1992). Two elements- - one that is tilted $X$ degrees in the clockwise direction, and one that is tilted $X$ degrees in the counterclockwise direction, with respect to their vertical orientation-are assumed to have equivalent saliency among elements with vertical orientation. Therefore, orientation is more useful than shape as a target-defining feature for controlling the saliency of a target.

\section{EXPERIMENT 1}

In Experiment 1, the effect of the extent of prior knowledge of a target feature value for overriding stimulusdriven interference was examined using a singleton distractor that had saliency equivalent to that of a target among distractors with respect to the target-defining feature. As a control condition, a simple feature search task in which the target was a green left-tilted rectangle and the nontarget was a green vertically oriented rectangle was used. This condition was referred to as the no-distractor 
Target present

(a)

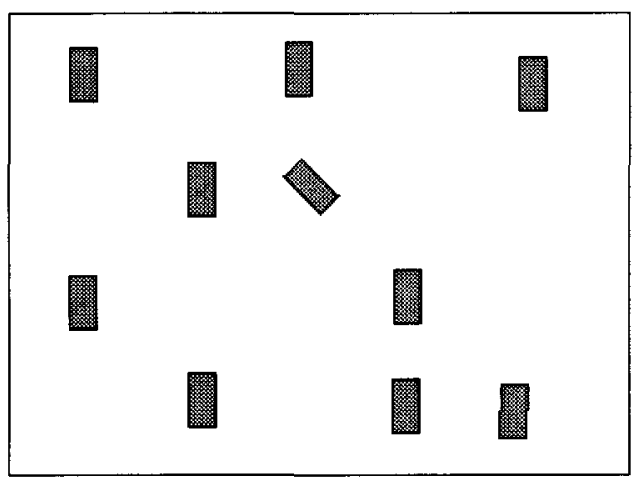

(b)

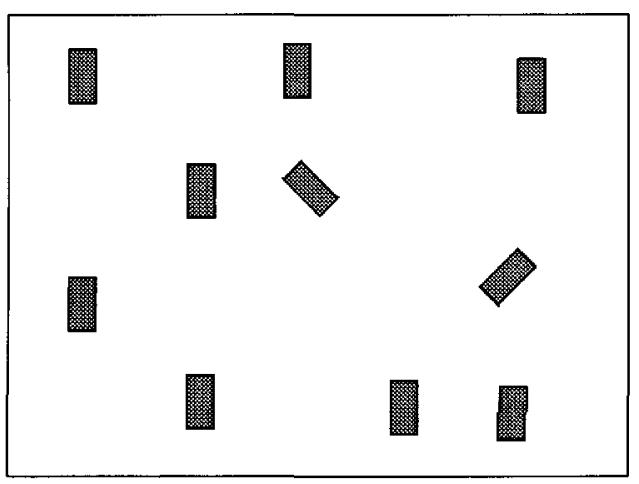

Target absent

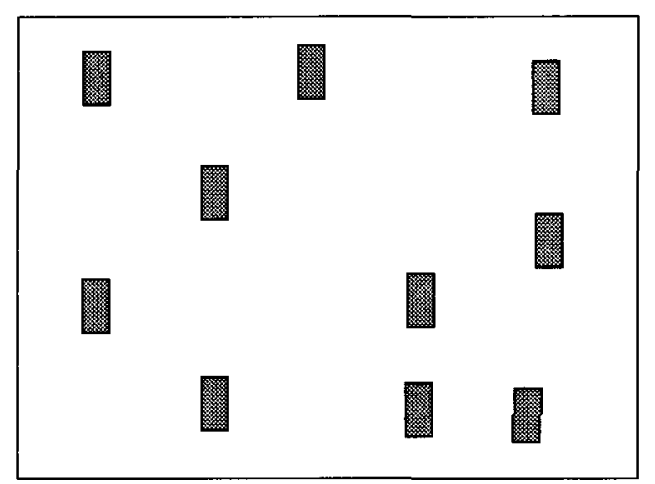

Figure 1. Examples of stimulus displays in Experiment 1: (a) no-distractor condition and (b) singletondistractor condition. Left panels show target-present trials; right panels show target-absent trials.

condition. The subjects' task was to search for the prespecified target and to respond to its presence or absence. In the singleton-distractor condition, one of the nontargets in the no-distractor condition was replaced with a singleton distractor. The singleton distractor was a green rectangle tilted to the same degree as the target, but in the opposite direction (i.e., to the right). Since both the target and the singleton distractor differed from the nontargets only in orientation, the stimulus-driven activation of the target and that of the singleton distractor were assumed to be equivalent on the orientation feature map. If knowledge of a specific target feature value (i.e., lefttilted in the present display) is available for overriding the stimulus-driven activation, it is expected that no interference from the singleton distractor will be observed.

\section{Method}

Subjects. Eight volunteers ( 4 males and 4 females; $21-26$ years of age) participated as paid subjects. All had normal or correctedto-normal visual acuity and normal color vision.
Apparatus and Stimuli. Visual stimuli were generated and controlled by a microcomputer (Apple Macintosh IIsi) and were presented on a 13-in. color CRT display (Apple Color High-Resolution RGB Monitor). The display elements were green (CIE $x, y$ coordinates of $\left..258 / .479 ; 17.58 \mathrm{~cd} / \mathrm{m}^{2}\right)$ rectangles $\left(10 \times 5 \mathrm{~mm} ; 1^{\circ} \times 0.5^{\circ}\right.$ in visual angle). Two singleton-distractor conditions were presented. First, in the no-distractor condition (Figure 1a), the target was defined only by orientation; the target was a green rectangle tilted $45^{\circ}$ to the left from its vertical orientation, and the nontarget was a green vertically oriented rectangle. Under the singletondistractor condition (Figure 1b), one of the nontargets used under the no-distractor condition was replaced by a singleton distractor. The singleton distractor was a green rectangle tilted $45^{\circ}$ to the right from its vertical orientation. Under the two singleton-distractor conditions, there were three levels of display size defined by the total number of display elements: 5,10 , and 15 . The elements were randomly scattered in cells of an imaginary $4 \times 5$ matrix $(80 \times 100$ $\mathrm{mm} ; 8^{\circ} \times 10^{\circ}$ in visual angle). In half of the trials, the target was presented; in the other half, it was not. Trials to test the two conditions were each repeated in four separate blocks. Each block was composed of 120 ( 3 display sizes $\times 2$ responses $\times 20$ repetitions) trials presented in random order. The order of presentation of the eight blocks was counterbalanced among the subjects. Before the 


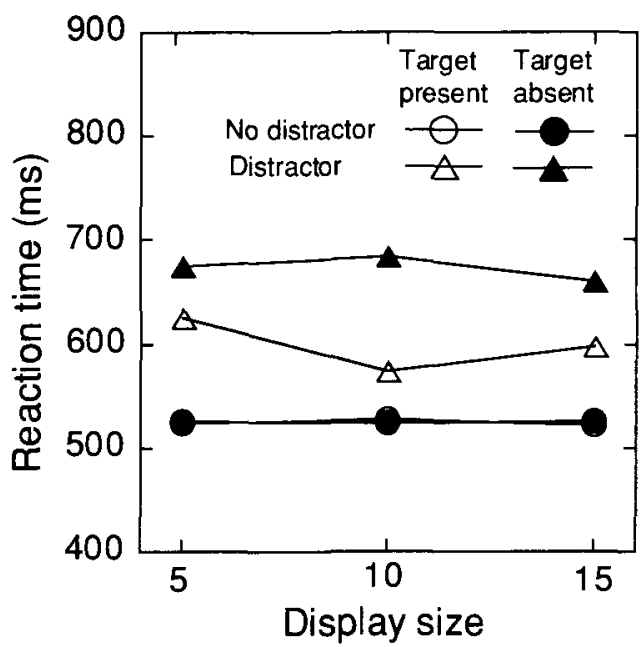

Figure 2. Mean correct RTs as a function of response and display size in Experiment 1.

presentation of the first block under each distractor condition, the subjects received a practice block (30 trials).

Procedure. Each subject was seated $57 \mathrm{~cm}$ from the CRT display, with his/her head supported by a chinrest. The sequence of each trial was as follows. First, a small white dot was presented as a fixation point in the center of the display for $2 \mathrm{sec}$. After $1 \mathrm{sec}$ of blank display, a visual search display was presented until the subject responded. The subject's task was to search for a prespecified target and to respond to the presence or absence of the target as quickly and accurately as possible by pressing one of two keys. The two keys were assigned to the index fingers of each hand. Half of the subjects pressed a key using the right index finger when the target was present and another key using the left index finger when it was absent, and this was reversed for the other half. The time from the onset of the presentation of the visual search display to the initiation of response was measured as the RT in each trial.

\section{Results}

In this experiment and all subsequent experiments reported in this article, the RT outliers were removed from the data set prior to analysis. The outliers were defined as RTs faster than $200 \mathrm{msec}$ or slower than $3 S D$ s above the mean for the subject and the condition. In Experiment 1 , this resulted in the removal of $2.47 \%$ of all observations.

The mean correct RTs are plotted as a function of display size in Figure 2. A three-way analysis of variance (ANOVA), with main terms of distractor condition, response, and display size, was performed. All of the main effects were significant [distractor condition, $F(1,7)=$ $47.08, p<.01$; response, $F(1,7)=14.91, p<.01$; display size, $F(2,14)=3.99, p<.05]$. All of the two-way interactions were also significant [distractor condition $\times$ response, $F(1,7)=12.49, p<.01$; response $\times$ display size, $F(2,14)=6.06, p<.05$; distractor condition $\times$ display size, $F(2,14)=5.20, p<.05]$. The three-way interaction was not significant. Simple effect analysis of the distractor condition $X$ response interaction revealed a significant simple effect of the distractor for both target-present trials $[F(1,14)=15.18, p<.0 \mathrm{H}]$ and target-absent trials
$[F(1,14)=59.08, p<.01]$. Simple effect analysis of the distractor condition $\times$ display size interaction revealed that the mean RTs in the distractor condition were different from those in the no-distractor condition at all levels of display size [Display Size $5, F(1,21)=55.67, p<.01$; Display Size $10, F(1,21)=37.78, p<.01$; Display Size 15, $F(1,21)=39.47, p<.01]$. These analyses confirmed the significant distraction effects of the singleton distractor.

The slope of the RT function was determined by linear regression analysis of the mean RTs. For target-present and target-absent trials, the slopes under the no-distractor condition were -0.25 and $-0.07 \mathrm{msec} / \mathrm{element}$, and those under the singleton-distractor condition were -2.73 and $-1.56 \mathrm{msec}$ /element, respectively.

Table 1 shows the error rates for each condition of Experiment 1 . The error rates were subjected to an arcsine transformation and entered into a three-way (distractor condition $\times$ response $\times$ display size) ANOVA. None of the main effects or interactions were significant $(p>.05)$. The error rates remained constant across all conditions, suggesting that the subjects did not tend to make a speedaccuracy tradeoff.

\section{Discussion}

In Experiment 1, the singleton distractor differed from the nontargets with respect to only one feature dimension (i.e., orientation). Furthermore, the target and the singleton distractor had equivalent saliency among the nontargets with respect to the target-defining feature. Nevertheless, the singleton distractor interfered with the detection of the target. The distraction effect was $75.2 \mathrm{msec}$ (averaged across three display sizes) for target-present trials. If the knowledge of the target feature value could give any advantage for overriding stimulus-driven interference, no distraction effect of the singleton distractor would have been found. However, the present results suggest that the knowledge of a target feature value is insufficient for overriding the stimulus-driven interference. Attentional control setting for a specific feature value appears impossible, at least in the present task.

The present results might be explained by applying a data-driven selection model to selection among salient elements defined by a feature dimension. According to this model, since the target and the singleton distractor had equivalent saliency in the orientation dimension, one of the two salient display elements was first selected by

Table 1

Error Rates (Percent) for Each Condition of Experiment 1 Display Size

\begin{tabular}{llll}
\cline { 2 - 3 } & 5 & 10 & 15 \\
\hline
\end{tabular}

No-Distractor Condition

\begin{tabular}{lccc} 
Target present & 2.3 & 1.4 & 2.5 \\
Target absent & 0.9 & 1.1 & 0.8 \\
\multicolumn{4}{c}{ Singleton-Distractor } \\
Condition \\
Target present & 2.5 & 1.8 & \\
Target absent & 2.7 & 2.2 & 2.2 \\
\hline
\end{tabular}




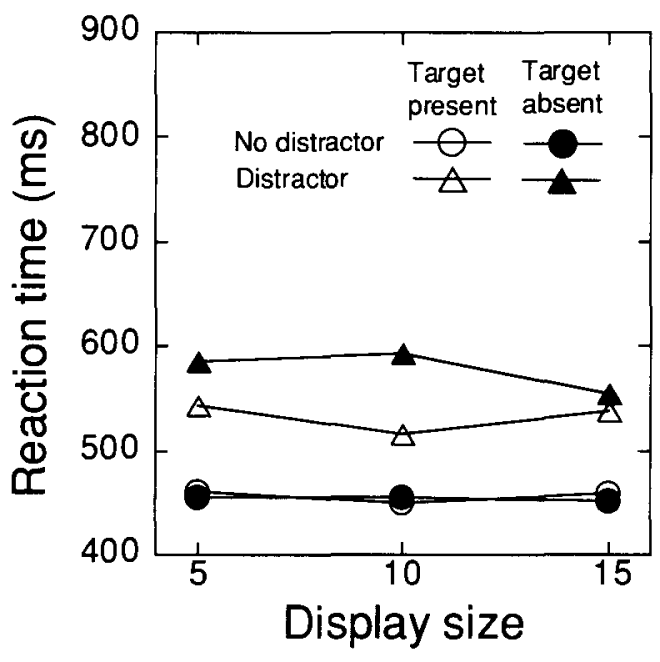

Figure 3. Mean correct RTs as a function of response and display size in Experiment 2.

chance. Therefore, the singleton distractor interfered with the detection of a target in the stimulus display of this experiment.

\section{EXPERIMENT 2}

In Experiment 1, knowledge of the target feature value was not enough to override stimulus-driven interference even though the target had saliency equivalent to that of the singleton distractor among nontargets. This result is consistent with the data-driven selection model. Experiment 2 was designed to test the validity of the data-driven selection model under within-dimensional selection. In this experiment, while the target and the nontarget were identical, just as in Experiment 1, the angular difference between the singleton distractor and the nontarget was reduced. This modification was intended to reduce the saliency of the singleton distractor with respect to the target-defining feature. It is known that the physical similarity between a target and a distractor in one feature dimension correlates with the efficiency of visual search (Duncan \& Humphreys, 1989). Furthermore, the physical similarity between two elements in a feature dimension is considered to be parallel to the saliency (Theeuwes, 1991a). Therefore, by modifying the orientation of the singleton distractor, a target is assumed to be more salient than the singleton distractor in a target-defining feature dimension. If the singleton distractor captures attention on the basis of this saliency, as suggested in the data-driven selection model, a less salient singleton distractor in the display would not capture attention in this experiment.

\section{Method}

Subjects. Eight new volunteers (6 males and 2 females; 20-33 years of age) participated as paid subjects. All had normal or corrected-to-normal visual acuity and normal color vision.

Apparatus and stimuli. The apparatus and the stimuli were identical to those in Experiment 1, except for the singleton distrac- tor. The singleton distractor was a green rectangle tilted $22.5^{\circ}$ to the right from its vertical orientation. ${ }^{1}$

Procedure. The task was identical to that described in Experiment 1.

\section{Results}

The RT outliers were discarded using the same procedure as in Experiment 1. In Experiment 2, this resulted in the removal of $3.13 \%$ of all observations.

The mean correct RTs are plotted as a function of display size in Figure 3. A three-way ANOVA, with the main terms of distractor condition, response, and display size, was performed. The main effects of distractor condition and display size were significant [distractor condition, $F(1,7)=40.03, p<.01$; display size, $F(2,14)=4.20, p<$ $.05]$, whereas the main effect of response was not significant. Two of the two-way interactions were also significant [distractor condition $\times$ response, $F(1,7)=8.88, p<$ .05 ; response $\times$ display size, $F(2,14)=11.18, p<.01]$. The distractor condition $\times$ display size interaction was not significant. The three-way interaction was significant $[F(2,14)=4.11, p<.05]$. Simple interaction analysis was conducted to reveal the effect of the distractor condition. The three-way interaction was decomposed on the basis of response. For target-present trials, the simple distractor condition $\times$ display size interaction was not significant $(p>.05)$, whereas there were significant simple main effects of distractor condition $[F(1,14)=18.58$, $p<.01]$ and display size $[F(2,28)=6.92, p<.01]$. For target-absent trials, the simple distractor condition $\times$ display size interaction was significant $[F(2,28)=6.19$, $p<.01]$. For target-present trials, the presentation of a singleton distractor prolonged the RTs.

The slope of the RT function was determined by linear regression analysis of the mean RTs. For target-present and target-absent trials, the slopes for the no-distractor condition were -0.25 and $-0.34 \mathrm{msec} / \mathrm{element}$, and those for the singleton-distractor condition were -0.47 and $-3.15 \mathrm{msec} / \mathrm{element}$, respectively.

Table 2 shows the error rates for each condition of Experiment 2. Error rates were subjected to an arcsine transformation and entered into a three-way (distractor condition $\times$ response $\times$ display size) ANOVA. The main effects of distractor condition and display size were significant [distractor, $F(1,7)=7.03, p<.05$; display size, $F(2,14)=4.34, p<.05]$, whereas the main effects of response and two- and three-way interactions were not sig-

Table 2

Error Rates (Percent) for Each Condition of Experiment 2

\begin{tabular}{cccc}
\hline & \multicolumn{3}{c}{ Display Size } \\
\cline { 2 - 4 } Response & 5 & 10 & 15 \\
\hline & No-Distractor Condition & \\
Target present & 2.8 & 1.4 & 3.0 \\
Target absent & 1.4 & 1.6 & 0.3 \\
Singleton-Distractor Condition & \\
Target present & 5.2 & 1.9 & 1.7 \\
Target absent & 3.1 & 2.8 & 2.3 \\
\hline
\end{tabular}


nificant. The significant main effects were due to the subjects' tendency of making many errors in the singletondistractor condition and in the 5-element trials. These cells showed rather slow RTs in Figure 3, suggesting that the subjects did not make a speed-accuracy tradeoff.

\section{Discussion}

These results showed a pattern similar to those found in Experiment 1. A less salient singleton distractor captured attention even when the subjects knew the target feature value. The distraction effect was $76.4 \mathrm{msec}$ for the target-present trials, which is very close to the effect size obtained in Experiment 1.

The distraction effect of a singleton distractor in Experiment 2 was statistically compared with that in Experiment 1. The RTs for target-present trials in both experiments were entered into a three-way ANOVA. The type of distractor $\left(45^{\circ}\right.$ tilt used in Experiment 1 or $22.5^{\circ}$ tilt in Experiment 2) was treated as a between-subjects factor. Distraction (no distractor or singleton distractor) and display size were treated as within-subject factors. The main effects of distraction and display size were significant [distraction, $F(1,14)=41.24, p<.01$; display size, $F(2,28)=13.91, p<.01]$, whereas the main effect of the type of distractor was not significant. Only the distraction $\times$ display size interaction was significant $[F(2,28)=$ $4.26, p<.05]$. The other two-way interactions and the three-way interaction were not significant. There was no significant effect of type of distractor or its interaction with other factors, suggesting that the distraction effect was not significantly reduced by the reduction of the saliency of a singleton distractor.

These data were incompatible with a model that assumed data-driven selection within a feature dimension, because the model predicted no distraction effect for a less salient singleton distractor.

\section{DISCUSSION OF EXPERIMENTS 1 AND 2}

The results of Experiments 1 and 2 revealed that knowledge of the target feature value was insufficient for overriding stimulus-driven interference. This finding was inconsistent with the prediction of the feature search mode of visual search (Bacon \& Egeth, 1994). Top-down control of visual attention for attending to a prespecified target feature value was impossible. Furthermore, the results of Experiment 2 indicated that a singleton distractor that was less salient than a target captured attention. Topdown control for a known feature value could not override the interference even when the singleton distractor was less salient than the target. This result suggests that the data-driven selection model (Theeuwes, 1991 a, 1992, 1994) could not be applied for selection within a feature dimension.

The results of these experiments are consistent with the hypothesis that attention is captured by any singletons that have unique feature values in a target-defining feature dimension. For the selection of a target on presentation with an irrelevant within-dimensional singleton distractor, prior knowledge of the target feature value or the data-driven saliency-based control cannot be used. Therefore, the present results suggest that all singletons within a specific feature dimension have the potential to capture attention.

One possible explanation for the results of Experiments 1 and 2 was based on the contingent involuntary orienting hypothesis of Folk and his colleagues (Folk, Remington, \& Johnston, 1992; Folk, Remington, \& Wright, 1994; see also Folk \& Remington, 1998). They argued that attentional capture can always be achieved by a variety of feature singletons when the singleton is contingent to the subject's attentional control settings. Singletons that are consistent with attentional settings will capture attention. They assumed that the discontinuities produced by a feature singleton could be distinguished into static discontinuities and dynamic discontinuities. If the subjects adopted their attentional setting for a static discontinuity (e.g., color), then any static discontinuity would capture attention. According to the contingent involuntary orienting hypothesis, the present results can be explained by assuming that the subjects adopted their attentional setting for static discontinuities. Then, since attentional setting is tuned to static discontinuities, including an orientation feature dimension, any discontinuity on the target feature dimension could capture attention.

\section{EXPERIMENT 3}

Experiment 3 was designed to examine the effectiveness of knowledge of a target feature for overriding stimulusdriven interference from a singleton distractor defined by a different feature dimension. The experiment would serve to examine the extent of the interference beyond the target-defining feature dimension. This experiment was similar to those performed by Theeuwes (1992), with modifications to match the stimuli and the paradigm to those used in Experiments 1 and 2. In Experiment 3, the target was defined by an orientation dimension. A color dimension was used as another target-defining feature. One became a target, and the other became a distractor, depending on the target condition.

The data-driven selection model predicts the one-way interference. Since the color singleton is more salient than the orientation singleton, ${ }^{2}$ a color distractor would interfere with the detection of an orientation target, and an orientation distractor would not interfere with the detection of a color target. On the other hand, if subjects tune their attentional settings to broad or static feature dimensions, regardless of the subjects' knowledge of the targetdefining feature dimension, a singleton distractor would interfere with detection of a target under both target conditions. 


\section{Color target}

(a)

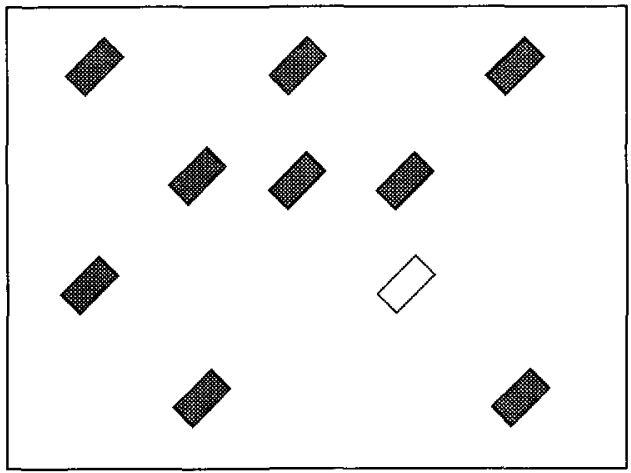

(b)

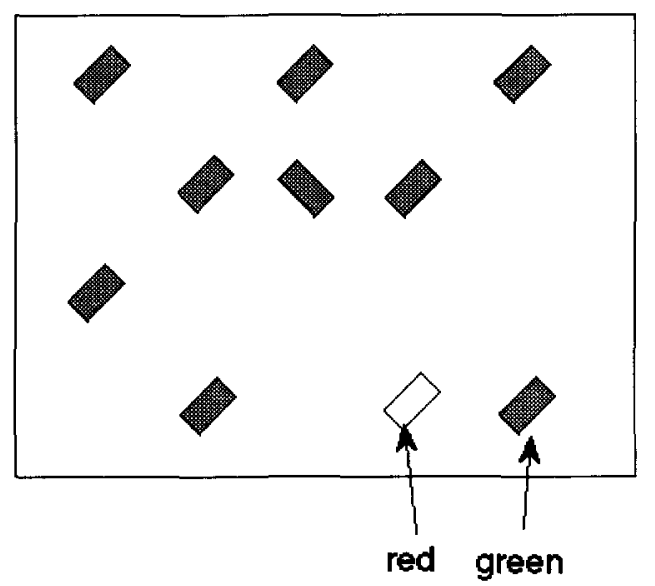

\section{Orientation target}

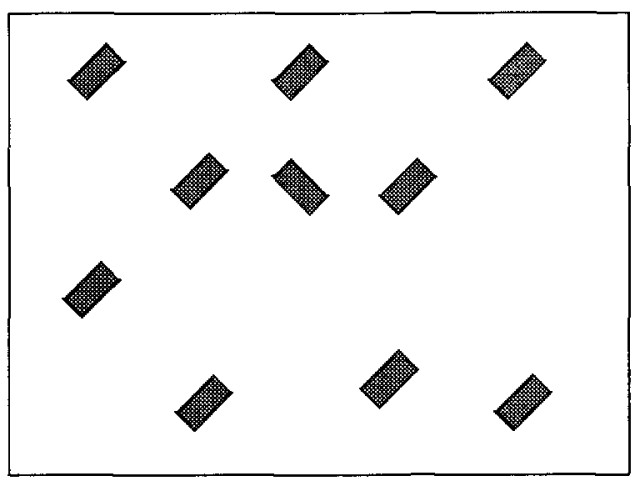

Figure 4. Examples of stimulus displays in Experiment 3: (a) no-distractor condition and (b) singletondistractor condition. Left panels show color-target condition; right panels show orientation-target condition.

\section{Method}

Subjects. Sixteen new volunteers (4 males and 12 females; 20-27 years of age) participated as paid subjects. The subjects were randomly assigned to two groups: one searching for an orientationdefined target, and the other searching for a color-defined target. All had normal or corrected-to-normal visual acuity and normal color vision.

Apparatus and Stimuli. The apparatus was identical to that in Experiment 1. The stimuli were identical to those in Experiment 1, except for the identities of the display elements. There were two target conditions: an orientation-target condition and a color-target condition. The display element was a rectangle of the same size as that used in Experiments 1 and 2. Under both target conditions, the nontargets were green rectangles tilted $45^{\circ}$ to the right from their vertical orientation. Under the color-target condition (left panels of Figure 4), two singleton-distractor conditions were presented. Under the no-distractor condition (Figure $4 a$ ), the target was a red (CIE $x, y$ coordinates of $.550 / .317 ; 11.78 \mathrm{~cd} / \mathrm{m}^{2}$ ) rectangle tilted $45^{\circ}$ to the right from its vertical orientation. Under the singletondistractor condition (Figure $4 \mathrm{~b}$ ), one of the nontargets used under the no-distractor condition was replaced by a singleton distractor. The singleton distractor was a green rectangle tilted $45^{\circ}$ to the left from the vertical orientation. Under the orientation-target condition (right panels of Figure 4), two singleton-distractor conditions were also presented. Under the no-distractor condition (Figure 4a), the target was a green rectangle tilted $45^{\circ}$ to the left from its vertical orientation. Under the singleton-distractor condition (Figure $4 b$ ), one of the nontargets used under the no-distractor condition was replaced by a singleton distractor that was a red rectangle tilted $45^{\circ}$ to the right from its vertical orientation. Thus, the red right-tilted rectangle and the green left-tilted rectangle exchanged roles as target or singleton distractor, under the color-target and orientation-target conditions.

The color-target condition and the orientation-target condition were designed as between-subjects conditions. Under each search condition, trials to test the two target conditions were each repeated in four separate blocks. Each block was composed of 120 (3 display sizes $\times 2$ responses $\times 20$ repetitions) trials presented in random order. The order of presentation of the eight blocks was counterbalanced among the subjects. Before the presentation of the first block of each distractor condition, the subjects received a practice block ( 30 trials).

Procedure. The task was identical to that described in Experiment 1. 
(a)

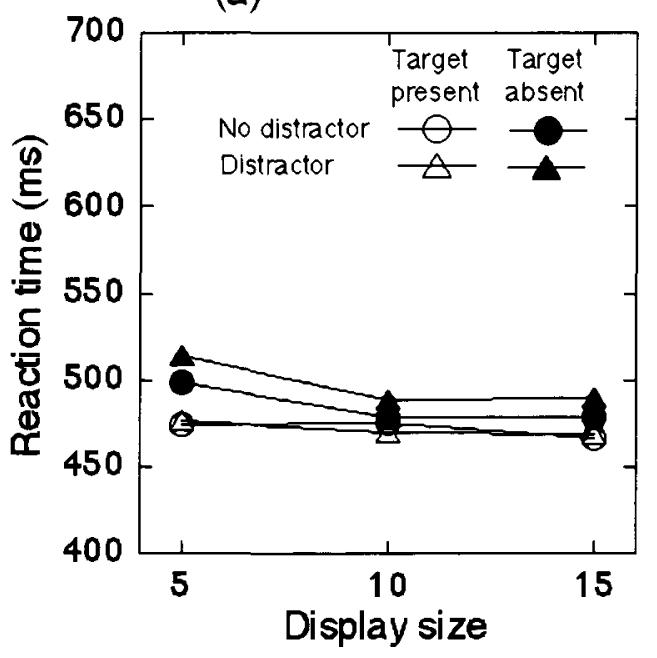

(b)

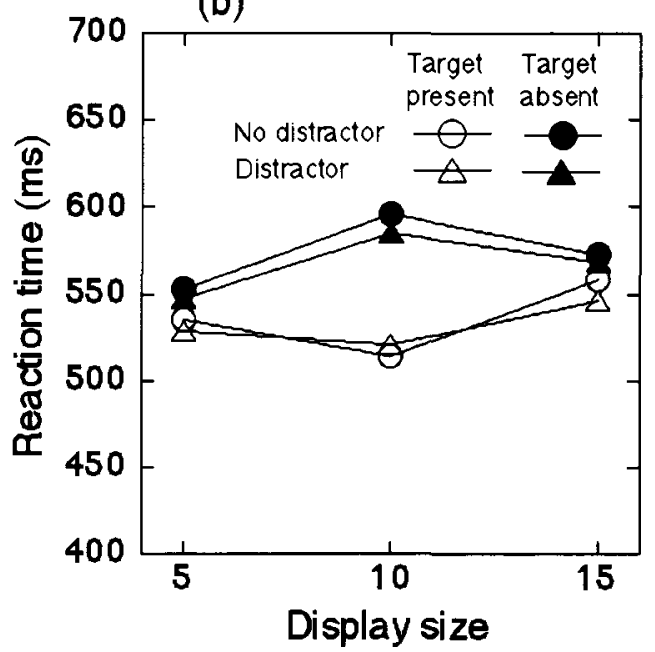

Figure 5. Mean correct RTs as a function of response and display size in Experiment 3: (a) color-target condition and (b) orientation-target condition.

\section{Results}

The RT outliers were discarded using the same procedure as that in Experiment 1. In Experiment 3, this resulted in the removal of $2.33 \%$ of all observations.

Color-target condition. The mean correct RTs for the color-target condition are plotted as a function of display size in Figure 5a. The mean RTs were subjected to a threeway ANOVA, with the main terms of singleton-distractor condition (no distractor and singleton distractor), response, and display size. The main effect of display size was significant $[F(2,14)=6.18, p<.05]$, whereas the main effects of distractor and display size were not significant $(p>05)$. There was a significant response $\times$ display size interaction $[F(2,14)=8.02, p<.01]$. The other two-way interactions and the three-way interaction were not significant. The result that there was no significant main effect of the distractor condition or of the interaction of the distractor condition and other factors suggests that the singleton distractor had no effect on the detection of a target.

To determine the slope of the RT function, the mean RTs were subjected to linear regression analysis. For targetpresent and target-absent trials, the slopes under the nodistractor condition were -0.76 and $-2.01 \mathrm{msec} / \mathrm{element}$, and those under the singleton-distractor condition were -0.85 and $-2.41 \mathrm{msec} / \mathrm{element}$, respectively.

The upper part of Table 3 shows the error rates for each condition of the color-target condition of Experiment 3. The error rates were subjected to an arcsine transformation and entered into a three-way (distractor condition $X$ response $\times$ display size) ANOVA. None of the main effects or interactions was significant $(p>.05)$. The error rates remained constant under all conditions, suggesting that the subjects did not make a speed-accuracy tradeoff.

Orientation-target condition. The mean correct RTs for the orientation-target condition are plotted as a function of display size in Figure 5b. The mean RTs were subjected to a three-way ANOVA, with the main terms of singleton-distractor condition, response, and display size. None of the main effects were significant $(p>.05)$. There was a significant response $\times$ display size interaction $[F(2,14)=6.63, p<.01]$. The other two-way interactions and the three-way interaction were not significant. Similarly to the case of the color-target condition, the result that there was no significant main effect of the distractor condition or of the interaction of the distractor condition and other factors suggests that the singleton distractor had no effect on the detection of a target.

To determine the slope of the RT function, the mean RTs were subjected to linear regression analysis. For target-present and target-absent trials, the slopes under the no-distractor condition were 2.33 and $2.01 \mathrm{msec} / \mathrm{element}$, and those under the distractor condition were 1.75 and $2.08 \mathrm{msec} / \mathrm{element}$, respectively.

Table 3

Error Rates (Percent) for Each Condition of Experiment 3

\begin{tabular}{ccc}
\multicolumn{3}{c}{ Display Size } \\
\hline 5 & 10 & 15
\end{tabular}

Color-Target Condition

No distractor

$\begin{array}{llll}\text { Target present } & 1.1 & 1.1 & 1.6 \\ \text { Target absent } & 0.8 & 1.3 & 1.4 \\ \text { Singleton distractor } & & & \\ \text { Target present } & 1.6 & 1.3 & 2.0 \\ \text { Target absent } & 1.1 & 1.1 & 0.6\end{array}$

Orientation-Target Condition

No distractor

Target present

Target absent

2.5

2.5

1.5

1.7

3.8

Singleton distractor

Target present

3.1

2.5 
The lower part of Table 3 shows the error rates for each condition of the orientation-target condition of Experiment 3 . The error rates were subjected to an arcsine transformation and entered into a three-way (distractor condition $\times$ response $\times$ display size) ANOVA. None of the main effects or interactions were significant $(p>$ .05 ). The error rates remained constant under all conditions, suggesting that the subjects did not make a speedaccuracy tradeoff.

\section{Discussion}

Under both target conditions, the patterns of the results were identical. There was no significant effect of the singleton distractor on detecting a target. The results were consistent with the predictions based on the feature search model. The subjects could use their knowledge of a target feature to override a stimulus-driven interference from an irrelevant feature dimension.

The results did not confirm the notion that attentional control settings are limited to static versus dynamic discontinuities, which was claimed by the contingent involuntary orienting hypothesis. The present results revealed that the subjects could adopt narrow attentional settings for a particular feature dimension. The results, however, do not imply that the subjects cannot extend their attentional settings for broad or several feature dimensions. In other words, the present results do not eliminate the possibility that a subject can attend to multiple feature dimensions when this strategy is the most adequate for solving the task. Recently, Folk and his colleagues argued that the setting of attentional control is flexible, depending on the constraints of the current tasks (Folk \& Annett, 1994; Folk \& Remington, 1996). They admitted that the feature search mode and the singleton detection mode showed a flexible state of attentional settings. However, further examinations are required to explore the limitations of this flexibility. The present results provided evidence for the limitations of the flexibility of attentional settings, in that subjects cannot adopt attentional control settings for a specific feature value.

The present results were inconsistent with those predicted from the data-driven selection model (Theeuwes, 1991a, 1992). The distraction effect of a color-defined singleton distractor on the detection of an orientationdefined target was expected. However, the present results showed the effect of a singleton distractor in neither the color-target condition nor the orientation-target condition, suggesting that the subjects might not have used the singleton detection strategy in the present experiment. The apparent difference between the results of Theeuwes and those of the present experiment might be due to the difference in the tasks assigned. The task used in the present experiment could be solved by detecting a discontinuity in the target-defining feature dimension. A singleton distractor might be filtered out in the preattentive level of processing. Furthermore, as Theeuwes (1992) suggested, since he used a compound search task, there was a clear separation of the perceptual process from the response process. In other words, there is no clear separation between them in the simple search task. Therefore, there remains a possibility that the series of results obtained in Experiments $1-3$ is specific for a simple visual search task, where employment of attention is not necessary for searching for a target. The next three experiments were intended to examine the within-dimensional and crossdimensional selectivity using a compound search task. Comparison between the results obtained using a simple visual search task and those obtained using a compound search task would enable a discussion on the locus of interference.

\section{EXPERIMENT 4}

In Experiments 4-6, a target and a singleton distractor were defined by their color or orientation. A reported feature that was independent of the target-defining feature was the relative location of a line segment intersecting a target. In Experiment 4, the within-dimensional interference effect obtained in Experiment 1 was examined using the compound search task. Identical to Experiment 1 , the target and the singleton distractor had equivalent deviation from a nontarget, but they had different feature values. In Experiment 1, the singleton distractor interfered with the detection of the target.

\section{Method}

Subjects. Eight new volunteers ( 2 males and 6 females; $21-25$ years of age) participated as paid subjects. All had normal or corrected-to-normal visual acuity and normal color vision.

Apparatus. The apparatus was identical to that of Experiment 1.

Stimuli and Procedure. The display element was composed of a green open rectangle $\left(15 \times 6 \mathrm{~mm} ; 1.5^{\circ} \times 0.6^{\circ}\right)$ and a line that horizontally intersected the rectangle by $1 / 3$ or $2 / 3$ position from the top of the rectangle (see Figure 6). There were three levels of display size defined by the total number of display elements: 5,10 , and 15 . The elements were randomly scattered in cells of an imaginary $4 \times$ 5 matrix $\left(80 \times 100 \mathrm{~mm} ; 8^{\circ} \times 10^{\circ}\right.$ in visual angle $)$.

Two types of tasks were used: a compound search task and a control task. In the compound search task, two singleton-distractor conditions (no distractor and distractor) were presented. In the nodistractor condition (Figure 6a), the target was defined by orientation; the target was a green rectangle tilted $45^{\circ}$ to the left from its vertical orientation, and the nontarget was a green vertically oriented rectangle. Under the singleton-distractor condition (Figure $6 \mathrm{~b}$ ), one of the nontargets used under the no-distractor condition was replaced by a singleton distractor. The singleton distractor was a green rectangle tilted $45^{\circ}$ to the right from its vertical orientation. Under the two singleton-distractor conditions, the target was presented in all trials. Each trial of the two singleton-distractor conditions was repeated in four separate blocks. Each block was composed of 96 ( 3 display sizes $\times 32$ repetitions) trials presented in random order. The order of presentation of the eight blocks was counterbalanced among the subjects. The first block of each condition was discarded as a practice block.

The procedure for the compound search task was identical to that described in Experiment 1, except for the subjects' task. The task 
(a)

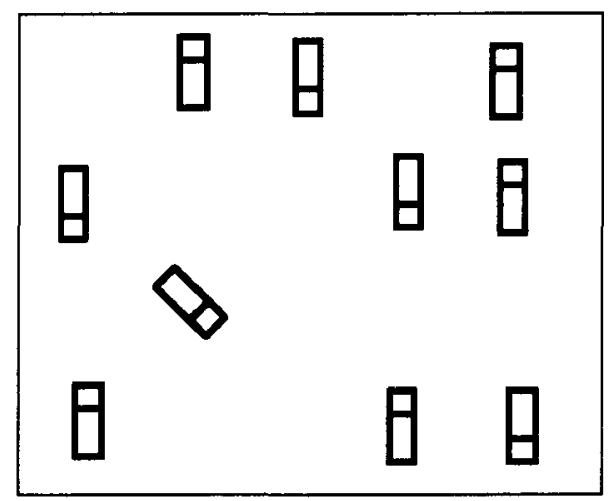

(b)

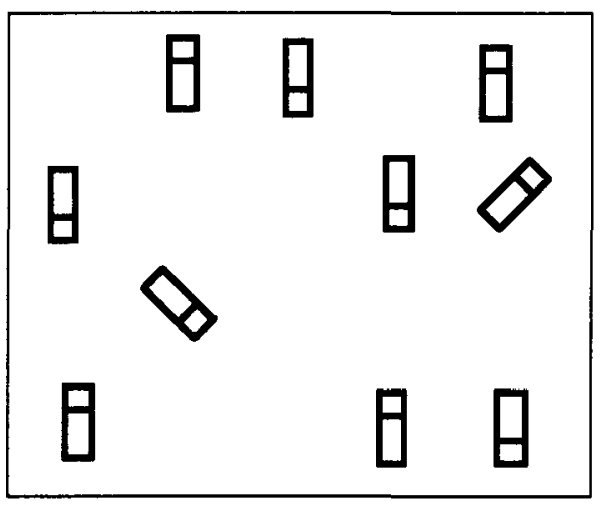

Figure 6. Examples of stimulus displays in Experiment 4: (a) no-distractor condition and (b) singletondistractor condition.

was to search for a prespecified target and to respond to the relative location of the line segment (upper or lower) intersecting the target as quickly and accurately as possible by pressing one of two keys. The two keys were assigned to the index fingers of both hands: the left index finger to indicate "upper," and the right to indicate "lower."

To ensure that the reported attribute (i.e., the relative location of a line segment in an open rectangle) was not available in parallel across a display, the visual search performance for the reported attribute was examined as a control task. In the control task, all display elements were vertically oriented rectangles. The target was defined by the relative location of the intersecting line segment; the target had a line segment in the upper part of a rectangle, and the nontarget had a line segment in the lower part of a rectangle. In half of the trials, the target was presented; in the other half, it was not. Trials of the control task were repeated in four blocks. Each block was composed of 96 ( 3 display sizes $\times 2$ responses $\times 16$ repeti- tions) trials presented in random order. The first block of trials was discarded as a practice block. The procedure for the control task was identical to that described in Experiment 1. The task was to search for a prespecified target and to respond to the presence or absence of the target as quickly and accurately as possible by pressing one of two keys.

All subjects participated in the two tasks; 4 subjects started off with four blocks of the control task, and the other 4 subjects started off with eight blocks of the compound search task.

\section{Results}

Control task. The RT outliers were discarded using the same procedure as that in Experiment 1. In the present task, this resulted in the removal of $0.85 \%$ of all observations. (a)

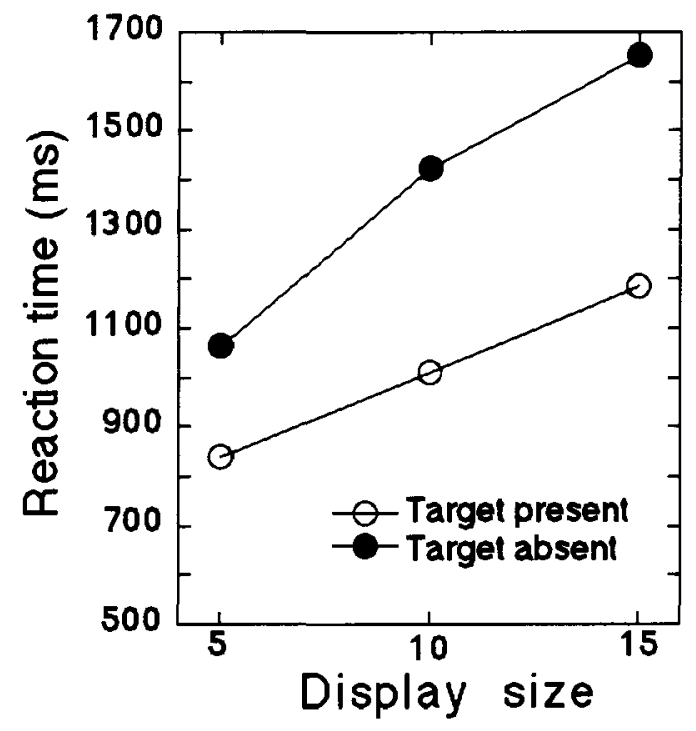

(b)

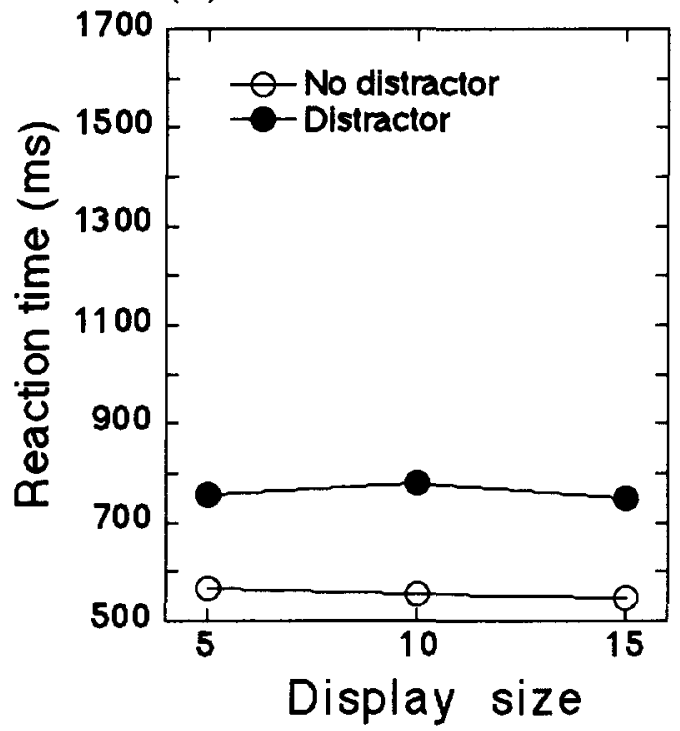

Figure 7. Mean correct RTs as a function of response and display size in Experiment 4: (a) control task and (b) compound search task 
Table 4

Error Rates (Percent) for Each Condition of Experiment 4

\begin{tabular}{lccc} 
& \multicolumn{3}{c}{ Display Size } \\
\cline { 2 - 4 } & 5 & 10 & 15 \\
\hline & Compound Search Task & \\
No distractor & 1.7 & 1.8 & 1.4 \\
Distractor & 2.3 & 2.3 & 1.7 \\
& Visual Search Task & & \\
Target present & 4.0 & 7.9 & 12.3 \\
Target absent & 1.4 & 0.6 & 0.8 \\
\hline
\end{tabular}

The mean correct RTs are plotted as a function of display size in Figure 7a. A two-way ANOVA, with the main terms of response and display size, was performed. The main effects of response and display size were significant [response, $F(1,7)=46.08, p<.01$; display size, $F(2,14)=106.22, p<.01]$. The response $\times$ display size interaction was also significant $[F(2,14)=18.08, p<.01]$.

The slope of the RT function was determined by linear regression analysis of the mean RTs. The slope for target-present trials was $34.80 \mathrm{msec} / \mathrm{element}\left(r^{2}=1.00\right)$, and that for target-absent trials was $58.56 \mathrm{msec} / \mathrm{element}$ $\left(r^{2}=.99\right)$.

Table 4 shows the error rates for each condition of the task. Error rates were subjected to an arcsine transformation and entered into a two-way (response $\times$ display size) ANOVA. The main effects of response and display size were significant [response, $F(1,7)=6.86, p<.05$; display size, $F(2,14)=8.28, p<.01]$. The response' $\times$ display size interaction was significant $[F(2,14)=16.36$, $p<.01]$. The error rates in target-absent trials were held constant across display sizes, whereas the error rates in target-present trials increased as a function of the display size. Neither tendency could be attributed to a speedaccuracy tradeoff.

Compound search task. The RT outliers were discarded using the same procedure as that in Experiment 1. In the present task, this resulted in the removal of $2.04 \%$ of all observations.

The mean correct RTs are plotted as a function of display size in Figure 7b. A two-way ANOVA, with the main terms of distractor condition and display size, was performed. The main effect of the distractor condition was significant $[F(1,7)=103.41, p<.01]$, whereas the main effects of display size and a two-way interaction were not significant.

The slope of the RT function was determined by linear regression analysis of the mean RTs. The slope for the no-distractor condition was $-1.92 \mathrm{msec} / \mathrm{element}$, and that for the distractor condition was $-0.62 \mathrm{msec} /$ element.

Table 4 shows the error rates for each condition of the task. Error rates were subjected to an arcsine transformation and entered into a two-way (distractor condition $x$ display size) ANOVA. None of the main effects or interactions was significant $(p>.05)$. The error rates remained constant under all conditions, suggesting that the subjects did not make a speed-accuracy tradeoff.

\section{Discussion}

The results of the control task clearly showed that acquiring information concerning the relative location of a line segment intersecting a rectangle required focal attention. This ensured that the reported attribute could not be accessed preattentively even though the target had a unique location of the line segment among nontargets. Therefore, focal attention was always needed to perform the compound search task.

In Experiment 4, the results of the compound search task replicated those obtained in Experiment 1. The distraction effect was $204.80 \mathrm{msec}$, averaged across the three display sizes. Knowledge of the target feature value was not available for overriding stimulus-driven interference even when the target had saliency equivalent to that of the singleton distractor among nontargets. This result was considered to be due to the limitation of attentional control setting for a specific feature value. The present results show that the limitation of attentional control setting is not confined to a simple search task, such as that used in Experiment 1.

\section{EXPERIMENT 5}

Experiment 5 was designed to examine the withinfeature interference using color dimension. One group of subjects searched for a green target among gray nontargets while ignoring a red singleton distractor. Another group of subjects searched for a red target among gray nontargets while ignoring a green singleton distractor. The purpose of Experiment 5 was to generalize the results obtained from the orientation dimension in Experiment 4 to the color dimension. There is evidence that the processing of orientation requires attentional resources (Braun $\&$ Sagi, 1990). Therefore, it would be valuable to show that the within-feature interference effect was not confined to the orientation dimension.

Furthermore, the two target conditions (i.e., a green target and a red target) served to further test the applicability of the data-driven selection model to the withindimensional selection. Assuming that one of the two targets is more salient than the other, this can be confirmed by comparing RTs under the no-distractor condition. Since the nontarget was the same in the two target conditions, the difference in RTs can reflect the relative saliency of the two targets among nontargets. If the singleton selection mode could be applied to the selection of an element among others within a feature dimension, presentation of a more salient singleton would interfere with the detection of a less salient singleton, whereas the less salient singleton would not interfere with the detection 


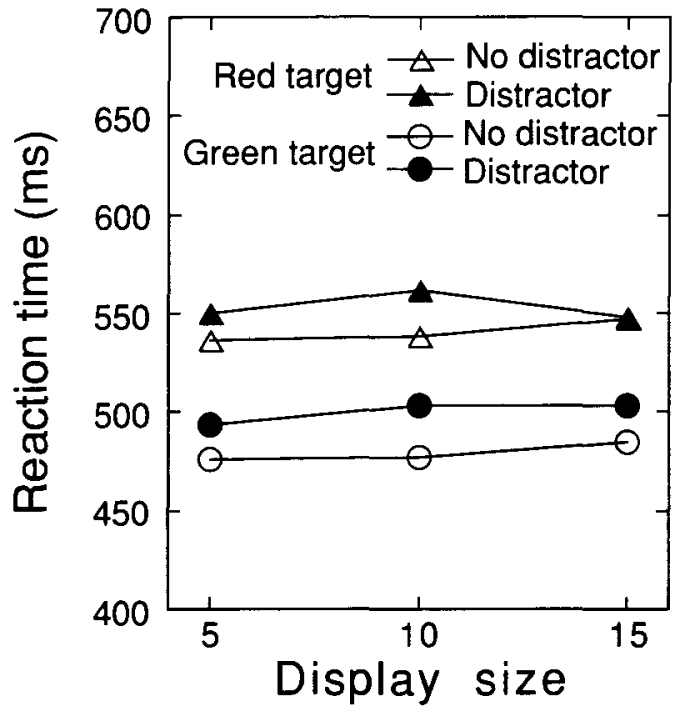

Figure 8. Mean correct RTs as a function of response and display size in Experiment 5.

of the more salient singleton. Such a one-way interference effect could be regarded as being consistent with the prediction from the data-driven selection model.

\section{Method}

Subjects. Sixteen new volunteers ( 8 males and 8 females; $19-25$ years of age) participated as paid subjects. The subjects were randomly assigned to two groups: one searching for a green target and the other searching for a red target. All had normal or corrected-tonormal visual acuity and normal color vision.

Apparatus and Stimuli. The apparatus and the stimuli were identical to those in Experiment 4, except for the display elements. All elements were vertically oriented open rectangles that differed in color. The nontarget was a gray rectangle $\left(10.53 \mathrm{~cd} / \mathrm{m}^{2}\right)$. In the green-target condition, the target was green and the singleton distractor was red. In the red-target condition, the target was red and the singleton distractor was green.

Procedure. The task was identical to that described in the compound search task in Experiment 4, except for the number of blocks. Trials to test the two conditions (distractor and no distractor) were each repeated in five separate blocks. The order of presentation of the 10 blocks was counterbalanced among the subjects. The first block of each condition was discarded as a practice block.

\section{Results}

Green-target condition. The RT outliers were discarded using the same procedure as that in Experiment 1. In the green-target condition, this resulted in the removal of $1.54 \%$ of all observations.

The mean correct RTs are plotted as a function of display size in Figure 8. A two-way ANOVA, with the main terms of distractor condition and display size, was performed. The main effects of distractor condition were significant $[F(1,7)=15.89, p<.01]$, whereas the main effects of display size and a two-way interaction were not significant.

The slope of the RT function was determined by linear regression analysis of the mean RTs. The slope for the no-distractor condition was $0.82 \mathrm{msec} / \mathrm{element}$, and that for the distractor condition was $0.96 \mathrm{msec} / \mathrm{element}$.

Table 5 shows the error rates for each condition of Experiment 5 . The error rates were subjected to an arcsine transformation and entered into a two-way (distractor condition $\times$ display size) ANOVA. None of the main effects or interactions was significant $(p>.05)$. The error rates remained constant under all conditions, suggesting that the subjects did not make a speed-accuracy tradeoff.

Red-target condition. The RT outliers were discarded using the same procedure as that in Experiment 1. In the red-target condition, this resulted in the removal of $1.74 \%$ of all observations.

The mean correct RTs are plotted as a function of display size in Figure 8. A two-way ANOVA, with the main terms of distractor condition and display size, was performed. The main effects of distractor condition were significant $[F(1,7)=9.01, p<.05]$, whereas the main effects of display size and a two-way interaction were not significant.

The slope of the RT function was determined by linear regression analysis of the mean RTs. The slope for the no-distractor condition was $1.06 \mathrm{msec} / \mathrm{element}$, and that for the distractor condition was $-0.17 \mathrm{msec} / \mathrm{element}$.

Table 5 shows the error rates for each condition of Experiment 5. Error rates were subjected to an arcsine transformation and entered into a two-way (distractor condition $X$ display size) ANOVA. None of the main effects or interactions were significant $(p>.05)$. The error rates remained constant under all conditions, suggesting that the subjects did not make a speed-accuracy tradeoff.

\section{Discussion}

The effect size of interference was relatively small but significant under both target conditions. The sizes of the distraction effect averaged across three display sizes were $21.2 \mathrm{msec}$ for the green-target condition and $12.3 \mathrm{msec}$ for the red-target condition. The distraction effects of the singleton distractor in each target condition were statistically compared. The RTs were entered into a three-way ANOVA. The target condition (green-target condition or red-target condition) was treated as a between-subjects factor. Distraction (no distractor or singleton distractor) and display size were treated as within-subject factors. The main effects of target condition and distraction were significant [target condition, $F(1,14)=8.14, p<.05$;

Table 5

Error Rates (Percent) for Each Condition of Experiment 5

\begin{tabular}{lcc} 
& Display Size \\
\hline 5 & 10 & 15
\end{tabular}

Green-Target Condition

$\begin{array}{lccc}\text { No distractor } & 2.4 & 3.0 & 2.0 \\ \text { Distractor } & 1.9 & 3.3 & 2.1 \\ & \text { Red-Target Condition } & & \\ \text { No distractor } & 2.3 & 2.4 & 1.9 \\ \text { Distractor } & 2.3 & 3.0 & 2.3\end{array}$




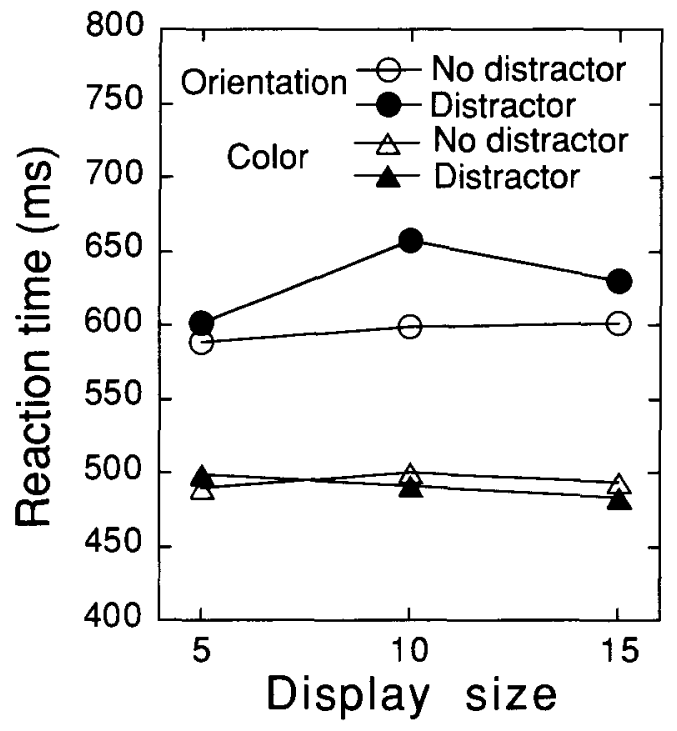

Figure 9. Mean correct RTs as a function of response and display size in Experiment 6.

distraction, $F(1,14)=24.89, p<.01]$, whereas the main effect of display size was not significant. Only the distraction $\times$ display size interaction was significant $[F(2,28)=3.926, p<.05]$. The other two-way interactions and the three-way interaction were not significant. The factor of the target condition did not significantly interact with that of the distraction, suggesting that the size of the distraction effect was not significantly different in the two target conditions.

Under both target conditions, a singleton distractor defined by a target-defining feature interfered with the detection of a target. Under no-distractor conditions, RTs in the green-target condition were faster than those in the red-target condition. Since there were no biases in assigning subjects into two target conditions, it seems reasonable to conclude that the green target was more salient than the red target among gray nontargets in the stimulus display used in Experiment 5 . If the data-driven selection model could be applied for the selection of a target within a color dimension, presentation of a green singleton distractor would interfere with the detection of a red target, whereas that of a red singleton distractor would not interfere with the detection of a green target. However, the present results were not consistent with such a prediction from the data-driven selection model.

The pattern of results obtained in Experiment 5 was consistent with that found in Experiment 2. A less salient singleton distractor can interfere with the detection of a more salient singleton target. These results show that the within-feature dimensional interference effect that occurred using the orientation dimension could also occur using the color dimension. These experiments showed that a two-way interaction was obtained under withindimensional selection irrespective of their stimulus di- mensions (orientation or color) and their tasks (simple search or compound search).

\section{EXPERIMENT 6}

In Experiment 6, cross-dimensional interference effects were examined using a compound search task. In Experiment 3, no cross-dimensional interference effect was found using a simple search task. This result was inconsistent with results of previous studies that showed one-way cross-dimensional interference (e.g., Theeuwes, 1991a). The most crucial difference between Experiment 3 and other experiments that reported crossdimensional interference was in the tasks used. The goal of Experiment 6 was to replicate the cross-dimensional interference effect using a compound search task with stimulus conditions comparable to that in Experiment 3.

\section{Method}

Subjects. Eight new volunteers ( 3 males and 5 females; $21-26$ years of age) participated as paid subjects. All had normal or corrected-to-normal visual acuity and normal color vision.

Apparatus and Stimuli. The apparatus and stimuli were identical to those of Experiment 4, except for the display elements. The design of the display elements emulated those in Experiment 3. Similar to Experiment 3, there were two target conditions: a colortarget condition and an orientation-target condition. Under both target conditions, the nontargets were green rectangles tilted $45^{\circ}$ to the right from their vertical orientation. Under the color-target condition, two singleton-distractor conditions were presented. Under the nodistractor condition, the target was a red rectangle tilted $45^{\circ}$ to the right from its vertical orientation. Under the singleton-distractor condition, a green rectangle tilted $45^{\circ}$ to the left from its vertical orientation was presented as a singleton distractor. Under the orientationtarget condition, two singleton-distractor conditions were presented. Under the no-distractor condition, the target was a green rectangle tilted $45^{\circ}$ to the left from its vertical orientation. Under the singletondistractor condition, a singleton distractor that was a red rectangle tilted $45^{\circ}$ to the right from its vertical orientation was presented.

Procedure. The task was identical to that described for the compound search task in Experiment 4, except for the number of blocks. All conditions were performed in a within-subjects design. For each target condition (color or orientation), trials to test the two conditions (distractor and no distractor) were each repeated in four separate blocks. The order of presentation of the eight blocks was counterbalanced among the subjects. The first block of each condition was discarded as a practice block. All subjects participated in the two target conditions; 4 subjects started off with eight blocks of the color-target condition, and the other 4 subjects started off with eight blocks of the orientation-target condition.

\section{Results}

The RT outliers were discarded using the same procedure as that in Experiment 1. In Experiment 6, this resulted in the removal of $2.03 \%$ of all observations.

The mean correct RTs are plotted as a function of display size in Figure 9. A three-way ANOVA, with the main terms of target condition, distractor condition, and display size, was performed. The main effects of target condition, distractor condition, and display size were significant [target condition, $F(1,7)=14.20, p<.01$; 
Table 6

Error Rates (Percent) for Each Condition of Experiment 6

\begin{tabular}{lccc}
\hline & \multicolumn{3}{c}{ Display Size } \\
\cline { 2 - 4 } & 5 & 10 & 15 \\
\hline & \multicolumn{3}{c}{ Color-Target Condition } \\
No distractor & 2.3 & 3.8 & 2.6 \\
Distractor & 3.1 & 2.9 & 3.3 \\
& Orientation-Target Condition & \\
No distractor & 1.2 & 2.9 & 2.9 \\
Distractor & 1.4 & 2.1 & 1.0 \\
\hline
\end{tabular}

distractor condition, $F(1,7)=6.68, p<.05$; display size, $F(2,14)=5.81, p<.05]$. Both two-way interactions were significant [target condition $\times$ distractor condition, $F(2,14)=9.75, p<.01$; distractor condition $\times$ display size, $F(2,14)=3.84, p<.05]$. Furthermore, the threeway interaction was significant $[F(2,14)=9.70, p<.05]$. The target condition $\times$ display size interaction was not significant. A simple interaction analysis was performed to reveal the effect of the distractor condition. The simple distractor condition $\times$ display size interaction was significant under the orientation-target condition $[F(2,28)=$ $19.05, p<.01]$, but not under the color-target condition. A simple simple effect of the distractor condition was found in 10-element trials and 15-element trials of the orientation-target condition $[F(1,42)=24.07, p<.01$, and $F(1,42)=5.89, p<.05$, respectively]. This result showed that the effect of a singleton distractor was significant only in 10- and 15-element trials under the orientationtarget condition.

The slope of the RT function was determined by linear regression analysis of the mean RTs. For the no-distractor condition and the distractor condition, the slopes under the color-target condition were 0.36 and $-1.64 \mathrm{msec} /$ element, and those under the orientation-target condition were 1.27 and $2.84 \mathrm{msec} /$ element, respectively.

Table 6 shows the error rates for each condition of Experiment 6 . Error rates were subjected to an arcsine transformation and entered into a three-way (target condition $\times$ distractor condition $\times$ display size) ANOVA. None of the main effects or interactions were significant $(p>.05)$. The error rates remained constant under all conditions, suggesting that the subjects did not make a speed-accuracy tradeoff.

\section{Discussion}

The color singleton distractor interfered significantly with target detection in the orientation-target condition, although there was no significant effect of the distraction in 5-element trials. However, the orientation singleton distractor did not interfere with the detection of the color target in any display-size condition. The pattern of the distraction effect of the singleton distractor replicated the results of Theeuwes (1991 a, 1992), which were obtained using color and form feature dimensions. These results suggest that the more salient element is selected irrespective of the knowledge of the target-defining feature dimension. This is consistent with results showing the data-driven selection model in cross-dimensional selection (Theeuwes, 1991a, 1992).

The present results were different from those in Experiment 3 . Experiment 3 showed complete crossdimensional selectivity based on knowledge of the targetdefining feature dimension when a simple search task was used. A similar interaction between the interference effect of an irrelevant distractor and the task required was reported by Kahneman, Treisman, and Burkell (1983). In one of their experiments, a target was defined by color and shape: The target was a white word, and each nontarget was a colored nonsense shape. Two different tasks were used. In an identification task, subjects read the target word aloud. In a detection task, subjects simply responded to the presence of the target. Kahneman et al. found that RT increased as a function of nontargets in the display under the identification task. However, RTs were independent of the number of nontargets in the detection task. They concluded that the irrelevant objects disrupts the deployment of attention in the limited-capacity stage when speeded identification is required. On the other hand, when detection of the target is required, the irrelevant objects do not interfere with processing of the target, because the response can be made on the basis of processing in the preattentive stage, without deployment of attention. Kahneman et al. referred to this disruptive effect of irrelevant objects occurred in the limited-capacity stage as filtering cost.

The differences in results obtained in Experiments 3 and 6 could be explained by the occurrence of the filtering cost. In the compound search task, deployment of attention to the target is required to extract the reported attribute from the target. Then the interference effect of the irrelevant and salient singleton distractor is inevitable. In other words, this result suggests that, when attention needs to be directed to the location of the target in order to respond, attention is guided solely by the salience of the elements in the display. This notion is consistent with the data-driven selection model. Therefore, it is possible to consider that the filtering cost occurs because feature level representation cannot be accessed in deployment of attention. On the other hand, in the case of a simple search task, when it is not necessary to direct attention to the location of the target, there is no interference from the irrelevant singleton distractor. Preattentive rejection of the

Table 7

Summary of Experimental Conditions and the Patterns of Interference in Experiments 1-6

\begin{tabular}{ccc}
\hline $\begin{array}{c}\text { Definition of a } \\
\text { Target and a Distractor }\end{array}$ & Simple Search & Compound Search \\
\hline Within-dimension & Two-way & Two-way \\
Cross-dimension & (Experiments I and 2) & (Experiments 4 and 5) \\
& No & One-way \\
(Experiment 3) & (Experiment 6) \\
\hline
\end{tabular}


irrelevant singleton distractor was observed when no shift of attention toward the target-defining attribute was required.

\section{GENERAL DISCUSSION}

Six experiments examined the limitations of knowledge of the target for overriding interference from an irrelevant feature singleton distractor. Two types of tasks, a simple search task (Experiments 1-3) and a compound search task (Experiments 4-6), were used. Two different definitions for a target and a singleton distractor were also used: In Experiments 1, 2, 4, and 5, a target and a singleton distractor were defined in the same feature dimension; in Experiments 3 and 6, they were defined in cross-dimensions. Table 7 summarizes the results of Experiments $1-6$ with respect to the pattern of interference from a singleton distractor as a function of the definition of the distractor and the task.

In within-dimension conditions, interference from a singleton distractor occurred in both single and compound search tasks, even when the singleton distractor was less salient than a target. On the other hand, in cross-dimension conditions, the singleton distractor interfered with the search for a target, only when the singleton distractor was more salient than the target, and the compound search task was used. When a simple search task was used, a singleton distractor did not interfere with the search for a target, irrespective of the relative saliency of the distractor to the target. Duncan (1985) suggested that the two types of tasks used in this study required different processes for a response: For the compound search tasks, the target-defining feature is analyzed in a first stage, which has little or no capacity limitation, whereas the reported attribute is analyzed in a second stage, which has substantial capacity limitation. For the simple search tasks, responses are based only on the processing of the first stage. In the cross-dimension conditions, the pattern of results varied between the two tasks; the interference of a singleton distractor especially occurred only in the compound search task, suggesting that the interference depended on the involvement of the second stage of processing. In contrast, in the within-dimension conditions, the type of task had no effect, suggesting that involvement of the second stage is not crucial for the interference.

With respect to cross-dimension conditions, Experiment 6 (in which the compound search task was used) replicates the results of previous studies (Theeuwes, 1991a, 1992). A salient singleton distractor interfered with the search for a target, whereas a less salient singleton distractor than a target did not interfere with the search for the target. The results are consistent with the data-driven selection model, in which a saliency between feature dimensions was used for the selection. However, when a simple search task was used, the interference effect was eliminated. This difference in the pattern of interference from an irrelevant distractor, between the simple reaction task and the choice reaction task, was known as the filtering cost (Kahneman et al., 1983). The failure of selection of a target with presentation of an irrelevant distractor in the choice reaction task was explained by the competitive effect of attentional employment between object files, which is episodic and temporary representations and is addressable by spatiotemporal coordinates and by physical attributes. However, this explanation is insufficient for interpreting the one-way interaction obtained in this study and previous studies in compound search tasks. To explain these results, some form of saliency should be computed between several feature dimensions or between objects files, and the relative saliency should be used for prioritizing in allocation of spatial attention (Koch \& Ullman, 1985; Müller et al., 1995; Theeuwes, 1991a, 1992).

In Experiment 3, when a simple search task was used in cross-dimension conditions, knowledge of a target feature dimension was useful in overriding the interference from a singleton distractor defined in a different feature dimension. This result is consistent with the prediction from the complete top-down selection model, whereas it is inconsistent with the data-driven selection model, although the results obtained in the compound search task are consistent with the latter model. The results of the cross-dimensional simple search task are also compatible with those in some models using simple search tasks, which suggest that the feature-processing stage can be modulated by top-down control. For example, in a revised version of the feature integration theory (FIT) of attention (Treisman \& Sato, 1990), a mechanism of top-down control is assumed, which serves to inhibit the excitation of particular feature maps that possess nontarget feature values. In their Guided Search model, Wolfe and his colleagues (Cave \& Wolfe, 1989; Wolfe, 1994) also assumed a mechanism of top-down control, which serves to excite a particular feature value on a feature map, resulting in the saliency on the activation map. Recently, Müller and his colleagues (Found \& Müller, 1996; Müller et al., 1995) proposed a dimension-weighting mechanism that serves to weight a specific feature dimension when a target feature is known. The total activation of each of the stimulus elements was computed in terms of the weighted sum of individual dimension-specific saliency maps. When the target feature is known, the feature dimension is assigned a large weight. With respect to the extent of top-down control, the present results are compatible with the dimension-weighting mechanism.

When attention employment to the target is not required, the top-down dimension weighting mechanism can be used for a search for the target. In other words, when the task is to decide the presence of a singleton target on a target-defining feature dimension (i.e., simple search task), a singleton distractor in another dimension can be ignored irrespective of the relative saliency of the target and the singleton distractor. On the other hand, when the subject must discriminate a target (i.e., compound search task), spatial attention must be employed for the location of the target. Then the top-down knowl- 
edge to the target-defining feature dimension is not available for the search of the target. Instead, the relative saliency between a target and a singleton distractor must be used. This pattern of results is consistent with the view that there are two main visual pathways; the occipitoparietal "where" pathway and the occipitotemporal "what" pathway (Ungerleider \& Mishkin, 1982). The dimension-weighting is assumed to be mediated by the weighted-dimension-related cortical areas (Found \& Müller, 1996). Color or orientation is considered to be processed mainly in some areas in the occipitotemporal pathway. On the other hand, parietal area is thought to play a role in the control of spatial attention (LaBerge, 1990). The results of Experiment 6 indicate that saliencybased control of spatial attention carried out in the parietal pathway is independent of the dimension-weighting computation concurrently running in the temporal pathway. Together with the results of Bacon and Egeth (1994), perhaps, the weighted dimensional signal can be used as the source of control of spatial attention only if the feature search mode is voluntarily selected by subjects. In a simple search task, since the deployment of spatial attention is not necessary for the search for a target, the detection of the target could be performed on the basis of the weighted dimensional signal in the temporal pathway.

The results of the experiments under cross-dimension conditions should be considered in terms of the limitations of top-down penetration to feature processing stage in controlling a spatial attention. According to the dimension-weighting account of Müller et al. (1995), saliency is computed in an individual dimension-specific saliency map, and the saliency map of an attended feature dimension is strongly weighted. Since the output of the dimension-specific saliency map is summed in the master map, saliency of stimulus-driven activation affects the detection of the target. This account does not agree with the present results obtained under the compound search task or the results consistent with the datadriven selection model. Müller et al. also pointed out these conflicts between their dimension-weighting accounts and the previous data and presented no solutions for the conflict. This study may provide a solution for the conflict; dimension-weighting output of the master map cannot be the source of the control of spatial attention. Although Müller et al. did not directly examine the effect of knowledge of the target-defining feature dimension in controlling the spatial attention, on the basis of the present results, it is predicted that dimensionally weighted saliency of a target is not effective when a compound search task rather than a visual search task is used. This prediction remains to be tested.

When a target and a singleton distractor were defined in the same feature dimension, both simple and compound search tasks showed the same pattern of results. The interference from a singleton distractor defined by the same feature as a target was inevitable when the saliency of the singleton distractor was equivalent to that of the target with respect to the target-defining feature
(Experiments 1 and 4) and even when a singleton distractor was less salient than a target (Experiments 2 and 5 ). These results could be interpreted as indicating that knowledge of a target feature value was not available for overriding the interference from a singleton distractor that was unique with respect to the target-defining feature. These results are inconsistent with both the datadriven selection model and the complete top-down selection model. Therefore, a new explanation is needed for these results.

Folk et al. (1992) in their Experiment 4 showed similar evidence of the limitations of top-down penetrability to a known feature value using a precue paradigm instead of visual search tasks. When a precue was defined by a color (green), the processing of a subsequently presented target defined by a different color (red) was disrupted by the precue. The disruption effect was similar to the result of the experiment in which a precue and a target were defined by the same feature value (i.e., red-red). This result is interpreted as converging evidence that subjects cannot attend to a particular feature value. However, recently, Folk and Remington (1998) found that the disruption effect of attentional capture could be distinguished in two forms: First, when the singleton distractor was defined by the same feature value as the target, substantial cost in terms of RT was obtained in the invalid distractor-location condition; second, the presentation of a feature singleton possessed of a different feature value from that of the target with respect to the target-defining feature showed an RT cost comparable to the condition in which no distractor was presented. Folk and Remington argued that the latter form of disruption is not attentional capture but the "filtering cost," because the effect size of the cost is consistent as a function of the spatial location of the target and the distractor (i.e., valid or invalid conditions). Interestingly, this nonspatial disruption effect seems to be compatible with the within-dimensional interference effect obtained in this study because the two-way interference between a red and a green singleton was obtained in both studies. In other words, the results of both studies showed that interference occurred irrespective of the relative saliency of the target and the singleton distractor. However, as Folk and Remington (1998) suggested, further examination is needed for clarifying the mechanism of these similar phenomena obtained in two different experimental paradigms.

Although top-down control on feature processing stages was assumed in several models of visual search, the extent of top-down control on feature level representation has not been clarified. A revised FIT (Treisman \& Sato, 1990) and the Guided Search model (Cave \& Wolfe, 1990; Wolfe, 1994) have difficulty in explaining the present results that showed the inability to exclude the interference from particular feature values, because these models assume top-down control on a specific feature value. Furthermore, the present results are consistent with the dimension-weighting mechanism (Found \& Müller, 1996; Müller et al., 1995) with respect to the ex- 
tent of top-down control. In light of the present results, the scope of dimension weighting is considered to be impossible to tune to a specific feature value, even when a singleton distractor is concurrently presented in a display.

Under the within-dimension conditions, another important issue is that there was no difference in the pattern of results from the two search tasks. Two hypothetical mechanisms may be suggested for the processing of within-dimensional target searching. First, there seems to be no doubt that spatial attention is employed in the location of a target in the compound search task. Therefore, the fact that a pattern of results similar to that in the compound search task was obtained in the simple search task suggests that attentional processes are involved in simple within-dimension search. Second, the information as to the within-dimensional saliency between a target and a singleton distractor is not used for the employment of the attention. Therefore, the results from the within-dimension condition suggest that there is another source of attentional control, which bypasses the saliency map. I argue that the computation of the saliency and the guidance of spatial attention were mediated by the occipitoparietal pathway. However, the present results indicate that there are several direct or indirect forms of occipitoparietal connections for controlling the spatial attention. In summary, the present study established a rule for within-dimensional selectivity that gives rise to limitations of top-down control on feature processes for overriding stimulus-driven interference. These limitations may reflect the impenetrability between processing modules belonging to two pathways, the occipitoparietal and occipitotemporal visual pathways.

\section{REFERENCES}

Bacon, W. F., \& EgETh, H. E. (1994). Overriding stimulus-driven attentional capture. Perception \& Psychophysics, 55, 485-496.

Braun, J., \& SAGI, D. (1990). Vision outside the focus of attention. Perception \& Psychophysics, 48, 45-58.

CAVE, K., \& Wolfe, J. M. (1990). Modeling the role of parallel processing in visual search. Cognitive Psychology, 22, 225-271.

DunCan, J. (1985). Visual search and visual attention. In M. I. Posner \& O. S. M. Marin (Eds.), Attention and performance XI (pp. 85-106). Hillsdale, NJ: Erlbaum.

Duncan, J., \& Humphreys, G. W. (1989). Visual search and stimulus similarity. Psychological Review, 96, 433-458.

Folk, C. L., \& ANNETT, S. (1994). Do locally defined feature discontinuities capture attention? Perception \& Psychophysics, 56, 277-287.

FolK, C. L., \& REmington, R. W. (1996). When knowledge does not help: Limitations on the flexibility of attentional control. In A. F. Kramer, M. G. H. Coles, \& G. D. Logan (Eds.), Converging operations in the study of visual selective attention (pp. 271-295). Washington DC: American Psychological Association.

Folk, C. L., \& Remington, R. W. (1998). Selectivity in distraction by irrelevant featural singletons: Evidence for two forms of attentional capture. Journal of Experimental Psychology: Human Perception \& Performance, 24, 847-858.

Folk, C. L., Remington, R. W., \& Johnston, J. C. (1992). Involuntary covert orienting is contingent on attentional control settings. Journal of Experimental Psychology: Human Perception \& Performance, 18, 1030-1044.

Folk, C. L., Remington, R. W., \& Johnston, J. C. (1993). Contingent attentional capture: A reply to Yantis (1993). Journal of Experimental Psychology: Human Perception \& Performance, 19, 682-685.

Folk, C. L., Remington, R. W., \& Wright, J. H. (1994). The structure of attentional control: Contingent attentional capture by apparent motion, abrupt onset, and color. Journal of Experimental Psychology: Human Perception \& Performance, 20, 317-329.

FouND, A., \& MüLLER, H. J. (1996). Searching for unknown feature targets on more than one dimension: Investigating a "dimensionweighting" account. Perception \& Psychophysics, 58, 88-101.

Kahneman, D., Treisman, A., \& Burkell, J. (1983). The cost of visual filtering. Journal of Experimental Psychology: Human Perception \& Performance, 9, 510-522.

Koch, C., \& UllmaN, S. (1985). Shifts in selective visual attention: Towards the underlying neural circuitry. Human Neurobiology, 4, 219 227.

LABERGE, D. (1990). Thalamic and cortical mechanisms of attention suggested by recent positron emission tomography experiments. Journal of Cognitive Neuroscience, 2, 358-372.

MÜller, H. J., Heller, D., \& Ziegler, J. (1995). Visual search for singleton feature targets within and across feature dimensions. Perception \& Psychophysics, 57, 1-17.

PAshler, H. (1988). Cross-dimensional interaction and texture segregation. Perception \& Psychophysics, 43, 307-318.

Theeuwes, J. (1991a). Cross-dimensional perceptual selectivity. Perception \& Psychophysics, 50, 184-193.

THEEUWES, J. (1991b). Exogenous and endogenous control of attention The effect of visual onsets and offsets. Perception \& Psychophysics, 49, 83-90.

Theeuwes, J. (1992). Perceptual selectivity for color and form. Perception \& Psychophysics, 51, 599-606.

THEEUWES, J. (1994). Stimulus-driven capture and attentional set: Selective search for color and visual abrupt onsets. Journal of Experimental Psychology: Human Perception \& Performance, 21, 799-806.

Treisman, A. (1988). Features and objects: The Fourteenth Bartlett Memorial Lecture. Quarterly Journal of Experimental Psychology, 40A, 201-237.

Treisman, A., \& Gelade, G. (1980). A feature integration theory of attention. Cognitive Psychology, 12, 97-136.

Treisman, A., \& Gormican, S. (1988). Feature analysis in early vision: Evidence from search asymmetries. Psychological Review, 95, 15-48.

Treisman, A., \& Sato, S. (1990). Conjunction search revisited. Journal of Experimental Psychology: Human Perception \& Performance, 16, 459-478.

Treisman, A., \& Souther, J. (1985). Search asymmetry: A diagnostic for preattentive processing of separable features. Journal of Experimental Psychology: General, 114, 285-309.

UNGERLEIDER, G. L., \& MishKin, M. (1982). Two cortical visual systems. In D. J. Ingle, M. A. Goodale, \& R. J. W. Mansfield (Eds.), Analysis of visual behavior (pp. 549-586). Cambridge, MA: MIT Press.

WolfE, J. M. (1994). Guided Search 2.0. A revised model of visual search. Psychonomic Bulletin \& Review, 1, 202-238.

WolfE, J. M., \& FRIEDMAN-HILL, S. R. (1992). Visual search for oriented lines: The role of angular relations between targets and distractors. Spatial Vision, 6, 199-207.

YANTIS, S. (1993). Stimulus-driven attentional capture and attentional control settings. Journal of Experimental Psychology: Human Perception \& Performance, 19, 676-681.

Yantis, S., \& Hillstrom, A. P. (1994). Stimulus-driven attentional capture: Evidence from equiluminant visual objects. Journal of Experimental Psychology: Human Perception \& Performance, 20, $95-$ 107.

Yantis, S., \& Johnson, D. N. (1990). Mechanisms of attentional priority. Journal of Experimental Psychology: Human Perception \& Performance, 16, 812-825.

YANTIS, S., \& JoNES, E. (1991). Mechanisms of attentional selection: Temporally modulated priority tags. Perception \& Psychophysics, 50, 166-178.

YANTIS, S., \& Jonides, J. (1990). Abrupt visual onsets and selective at- 
tention: Voluntary versus automatic allocation. Journal of Experimental Psychology: Human Perception \& Performance, 16, 121-134.

\section{NOTES}

1. A control experiment involving 8 subjects showed that search for a $45^{\circ}$ tilted rectangle among vertical rectangles was significantly faster (19.3 msec faster averaged across three display sizes) than that for a $22.5^{\circ}$ tilted rectangle in target-present trials. These results suggest that the $45^{\circ}$ tilted rectangle was more salient than the $22.5^{\circ}$ rectangle (see, e.g., Theeuwes, 1991 a, 1992).
2. A control experiment involving 8 subjects was performed using the two target conditions without the presentation of a singleton distractor. The results showed that search for a color target was $124.5 \mathrm{msec}$ faster (averaged across three display sizes) than that for an orientation target in target-present trials. These results suggest that the color target was more salient than the orientation target (see, e.g., Theeuwes, 1991a, 1992).

(Manuscript received December 21, 1995; revision accepted for publication December 27, 1997.) 